\title{
Optical emission from a charge-tunable quantum ring
}

\author{
R. J. Warburton ${ }^{1,2}$, C. Schäflein ${ }^{1}$, D. Haft ${ }^{1}$, F. Bickel ${ }^{1}$, A. Lorke ${ }^{1}$, K. Karrai ${ }^{1}$, J. M. Garcia ${ }^{3}$, W. \\ Schoenfeld ${ }^{3}$ and P. M. Petroff \\ Center for NanoScience and Sektion Physik, Ludwig-Maximilians-Universität, Geschwister-Scholl-Platz \\ 1, 80539 München, Germany \\ Materials Department and QUEST, University of California, Santa Barbara, California 93106 , USA \\ Present address: Department of Physics, Heriot-Watt University, Edinburgh EH14 4AS, UK
}

Correspondence to: R. J. Warburton ${ }^{1,2}$ Correspondence and requests for materials should be addressed to R.J.W. (email: Email:R.J.Warburton@hw.ac.uk

\begin{abstract}
Quantum dots or rings are artificial nanometre-sized clusters that confine electrons in all three directions. They can be fabricated in a semiconductor system by embedding an island of lowbandgap material in a sea of material with a higher bandgap. Quantum dots are often referred to as artificial atoms because, when filled sequentially with electrons, the charging energies are pronounced for particular electron numbers, ${ }^{1,2}, 3$; this is analogous to Hund's rules in atomic physics. But semiconductors also have a valence band with strong optical transitions to the conduction band. These transitions are the basis for the application of quantum dots as laser emitters $^{4}$, storage devices ${ }^{5,6,7}$ and fluorescence markers. ${ }^{8}$. Here we report how the optical emission (photoluminescence) of a single quantum ring changes as electrons are added oneby-one. We find that the emission energy changes abruptly whenever an electron is added to the artificial atom, and that the sizes of the jumps reveal a shell structure.

The nanometre-sized clusters for our experiments were made by self-assembly. We initially grew InAs quantum dots on GaAs by exploiting the strain-driven change from two-dimensional to three-dimensional growth which occurs after a coverage of 1.5 monolayers of InAs. A pause was introduced in the overgrowth with GaAs during which In migrated, causing the dots to become ring-like $e^{9,10}$. The rings have an $s$-like ground state and a $p$-like excited state, as for dots, so that the general features of shell filling are the same for both dots and rings. The Aharanov-Bohm effect, an interference of electron waves in a quantum ring, is not relevant here as we do not apply a magnetic field to generate a phase difference between clockwise and anticlockwise paths around the ring.
\end{abstract}

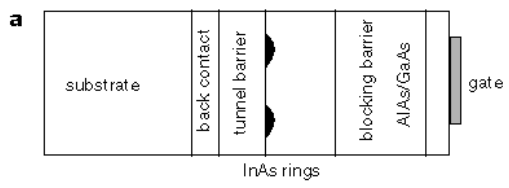

b

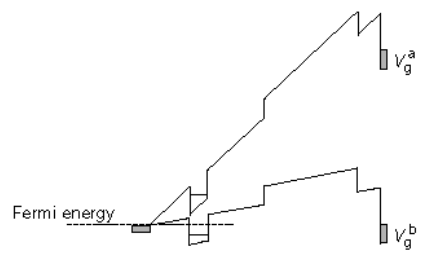

c

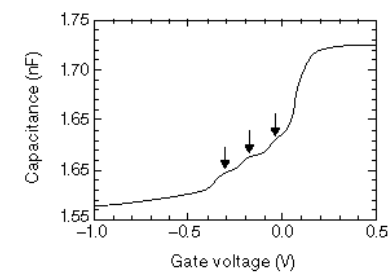

Figure 1 The semiconductor device for charging quantum rings with electrons. $\mathbf{a}_{1}$ The layer structure. The tunnel barrier is $25 \mathrm{~nm}$ thick, and the separation between back contact and surface is $175 \mathrm{~nm}, 30$ that a change in gate voltage $\Delta V_{g}$ implies a change in electrostatic potential (in eV) of $\Delta V_{9} / 7$. $b_{1}$ The band diagram at two different voltages, $V_{g}^{\mathrm{a}}$ and $V_{g}^{\mathrm{b}} . \mathrm{c}_{1}$ The capacitance of the device used for the optical experiments igate area $1.8 \mathrm{~mm}^{3}$ ) at $4.2 \mathrm{~K}$. The arrows mark three charging peaks.

We were faced with two experimental challenges. The first was to design a structure to load quantum rings sequentially with electrons. Our concept is to embed the InAs rings between highly doped GaAs and a surface gate electrode ${ }^{11}$, as shown in Fig. 1a. At large, negative gate voltages $\left(V_{g}\right.$ in Fig. $\left.1 b\right)$, the ring level lies well above the Fermi energy and is unoccupied. At a 
more positive $V_{g}$, the ring level is resonant with the Fermi energy and electrons tunnel into and out of the ring. A further increase in $V_{g}\left(V_{g}^{b}\right.$ in Fig. 1b) traps one electron in the ring. We can monitor the tunnelling by using the capacitance between gate and back contact ${ }^{12,}{ }^{13}$. Three charging peaks can be made out in the capacitance of a large ensemble of rings (Fig. 1c). The rise in capacitance at $V_{g}=0.1 \mathrm{~V}$ corresponds to tunnelling into the thin InAs layer between the rings (the wetting layer).

The second challenge was to measure a single ring. This is important as otherwise the 20-meV inhomogeneous broadening in the photoluminescence $(\mathrm{PL})$ obscures the shifts expected on charging. We measured PL with a low-temperature confocal microscope which has a diffractionlimited spatial resolution of $610 \mathrm{~nm}$ at a wavelength of $950 \mathrm{~nm}$. Our ring densities are $\approx 5 \times 10^{9}$ $\mathrm{cm}^{-2}$, implying that several tens of rings lie in the focus. Individual rings can be selected through their emission energy: we looked for rings in the low-energy tail of the spectral distribution. In order to investigate rings closer to the peak of the ensemble distribution, we increased the spatial resolution by defining 300-nm-sized holes in an aluminium film on the sample surface. Even in this case, we always detected emission from several rings. However, as each ring has unique charging voltages, the $V_{g}$ dependence of the PL allows us to determine which PL lines belong to which ring. We present here data from a sample without a metal mask as the signal/noise ratio is superior.

We recorded the PL as a function of $V_{\mathrm{g}}$. The data are represented as a colour-scale plot in Fig. 2. At $V_{\mathrm{g}} \approx-0.7 \mathrm{~V}$ there is a single, sharp peak which is the emission from a single ring. At $-0.6 \mathrm{~V}$ the PL energy decreases abruptly. The jump in energy occurs because an additional electron becomes trapped in the ring. This voltage, $-0.6 \mathrm{~V}$, is more negative than the first charging voltage of the ensemble average (Fig. 1C), and this correlates with the particularly low emission energy of the ring in Fig. 2. At higher $V_{g}$, further steps can be made out in the PL. In each case, a jump arises when an additional electron is added to the ring. The first jump at $-0.6 \mathrm{~V}, 6.0 \mathrm{meV}$ in energy, represents the binding energy of a singly charged exciton $\left(X^{{ }^{-}}\right)$. The second jump represents the energy needed to add an additional electron to the $\mathrm{X}^{1-}$ to form $\mathrm{X}^{2-}$, and so on. The first jump in energy is large, the second small, the third also small, and the fourth reasonably large. This is an optical manifestation of Hund's rules for electron charging. Whenever an electron can be added with the benefit of exchange energy, as is the case for the second and third steps, the charging energy is small. Whenever an electron is added to complete a sub-shell, the charging energy is large.

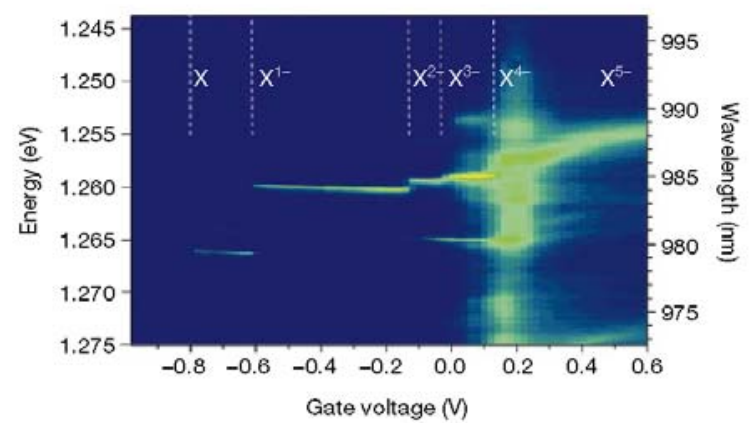

Figure $2 \mathrm{~A}$ colour-scale plot of the photoluminescence (PL) versus gate voltage at $4.2 \mathrm{~K}$. Here dark blue, green and yellow correspond to low, medium and high signals, respectively. PL was excited by generating electron-hole pairs in the wetting layer with a laser diode emitting at $822 \mathrm{~nm}$ wavelength. The pump power was kept below $1 \mu \mathrm{W}$ in order to populate the rings with no more than one exciton at a given time. The PL was dispersed with a 0.25-m grating spectrometer and detected with a cooled CCD camera, a set-up with spectral resolution $0.2 \mathrm{~nm}$. The main features are from a single ring. The PL at $1.265 \mathrm{eV}(980 \mathrm{~nm})$, however, pronounced around $0.0 \mathrm{~V}$, comes from a second ring.

On moving from $X^{1-}$ to $X^{2-}$, a satellite appears on the low-energy side of the main peak. The satellite can be just made out in the colour-scale plot, and is a clear feature in the individual spectra, a few of which are shown in Fig. 3. We can be confident that the two emission lines come from the same ring because the satellite emerges exactly when the PL changes from $X^{1-}$ to $\mathrm{X}^{2-}$ (see also Fig. 4). Also, the main line weakens at the transition but the total intensity stays constant to within $10 \%$. We claim that the $X^{2-}$ has two lines because there are two different final states $^{14}$, as shown in the level diagrams in Fig. 3. For $\mathrm{X}^{2-}$, the two possible final states have either parallel spins (a triplet state) or antiparallel spins (a singlet state). In exact analogy to the excited states of the helium atom, these two states are separated by twice the exchange 
energy, $2 X_{s p}$, and have degeneracies of 3 and 1 , respectively. We measure the splitting to be $3.6 \mathrm{meV}$, that is $X_{s p}=1.8 \mathrm{meV}$. The intensity ratio is $7 \pm 2: 1$, not the $3: 1$ expected from the degeneracies. This could be due to the electron-hole exchange interaction which tends to align the spins in the initial state, favouring the triplet final state ${ }^{15,16}$.
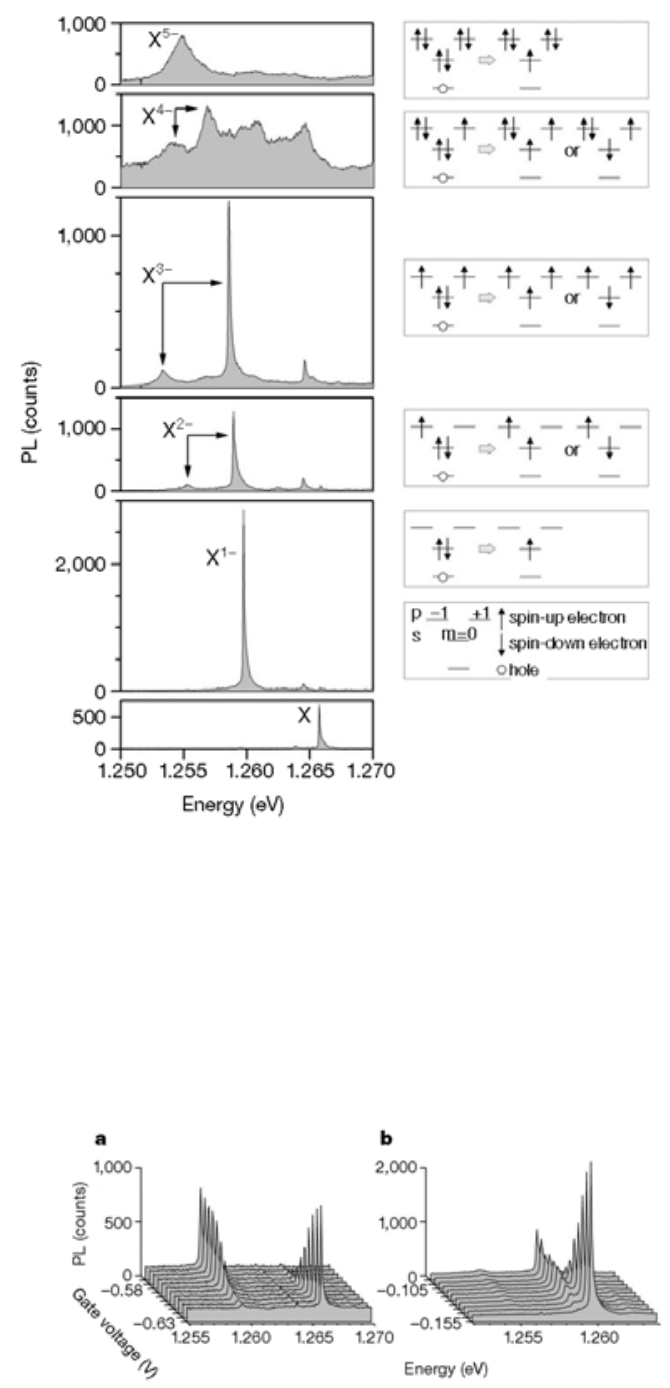

Figure 3 Photoluminescence from a single quantum ring for different charge states. Spectra are shown at $V_{g}=-0.76,-0.16,-0.10,0.40,0.22$ and $0.50 \mathrm{~V}$, corresponding to emission from the $X, X^{1-}, X^{2-}, X^{3-}, X^{4-}$ and $X^{5-}$ excitons, respectively. The PL red-shifts on charging, and a satellite occurs on the low-energy side of the main peak for $X^{2-}$ and $X^{3-}$. Note that the other, weaker emissions (for instance on the high-energy side of the main $X^{2-} \mathrm{PL}$ ) belong to another ring, and that the asymmetry in the sharp lines is an artefact of the spectrometer. The broad peak between the $X^{3-}$ main peak and the satellite may be emission from an excited initial state which recombines before it can relax. The level diagrams show the emission processes from initial state to possible final states. We: consider $s$ and $p$ states in the conduction band with angular momenta of 0 and \pm 1 , and the $s$ state in the valence band. In the experiment, both electrons and holes are unpolarized. To avoid redundancy in the figure, we sketch cases with electrons preferentially polarized up and an unpolarized hole.
Figure $4 \mathrm{PL}$ of a single quantum ring in the transition from one charge state to the next. $\mathbf{a}, X$ to $X^{1-} ; \mathbf{b}, X^{1-}$ to $X^{2-}$. Panel $\mathbf{b}$ also shows the emergence of the satellite at $1.255 \mathrm{eV}$.

For $\mathrm{X}^{3-}$ a similar argument applies; in this case the final state is analogous to the excited lithium atom. We have diagonalized the interaction matrix for three spin-1/2 electrons, and calculate a splitting of $3 X_{s p}$ and an intensity ratio 2:1. Experimentally, the splitting is 1.44 times larger than for the $X^{2-}$, remarkably close to the predicted 1.5 , and the satellite becomes more intense, 2 \pm 0.5 times weaker than the main peak. It is surprising that a treatment of the Coulomb interactions with perturbation theory works so well. This approach is justified when the electron and hole wavefunctions extend less than the excitonic Bohr radius, $10 \mathrm{~nm}$; yet here the circumference of the ring is $\approx 100 \mathrm{~nm}$. The $X^{3-}$ is the most complicated case; $X^{4-}$ is one electron short of a filled $p$ shell and should behave like $\mathrm{X}^{2-}$. In the data, the splitting for $\mathrm{X}^{4-}$ returns almost to the value for $\mathrm{X}^{2-}$ but the $\mathrm{PL}$ becomes very broad.

At large and negative $V_{g}$, the PL disappears; we interpret this as field ionization of the excitons. The electron tunnelling time, $\tau_{t}$, decreases rapidly with increasing $\left|V_{\mathrm{g}}\right|$, eventually becoming smaller than the recombination time, $\tau_{r}$. At a smaller $\left|V_{g}\right|\left(\tau_{t}>\tau_{r}\right)$, the 0 -electron and the 1electron states become degenerate and electron tunnelling occurs. We detect this as a jump in the PL energy. Figure 4 shows how the high-energy peak weakens and the low-energy peak 
strengthens with small steps in $V_{\mathrm{g}}$. It is puzzling that we observe both $\mathrm{X}$ and $\mathrm{X}^{1-}$ over a large range of $V_{g}$, some $20 \mathrm{mV}$, corresponding to a change in electrostatic energy of about $3 \mathrm{meV}$. This energy is much larger than the thermal energy $(0.36 \mathrm{meV})$. Heating from the laser is unlikely as an explanation, as broad single electron tunnelling peaks have been observed in transport experiments on similar samples ${ }^{17}$. The $3-m e V$ energy scale is also much larger than ring-ring interactions $(1 \mathrm{meV})$ which are significantly screened by the adjacent back contact ${ }^{13}$. To account for this result, we propose that there are temporal fluctuations in the potential (on a timescale large compared to $\tau_{t}$ ), caused by some sort of impurity state close to the ring. The effect is analogous to the spectral diffusion observed in the PL from single CdSe dots ${ }^{18}$. Figure 4 shows also the transition from $\mathrm{X}^{1-}$ to $\mathrm{X}^{2-}$, and this too shows a gradual cross-over from one line to the other. Given the 3-meV fluctuations, it is at first sight surprising that we see sharp PL lines at all. The explanation is that the PL energy is very sensitive to the charge in the ring, but only weakly sensitive to the electric field (almost flat plateaux in Fig. 2). The combination of the fluctuations with the slight $V_{\mathrm{g}}$-dependence of the $\mathrm{PL}$ energy constitutes a broadening mechanism. We predict this to be about $20 \mu \mathrm{eV}$ for our sample, comparable in fact to the expected homogeneous linewidth ${ }^{19}$.

For highly charged excitons, $\mathrm{X}^{2-}$ and above, the final state after emission of a photon is an excited state. In the simplest case, the ring emits a photon and then some time later relaxes into the ground state. But if the final state relaxes very quickly, the emission will be broadened by the energetic uncertainty of the final state. Therefore, we can use the PL linewidth as a measure of the relaxation rates of the final states. For $\mathrm{X}^{2-}$, the satellite is clearly broader (full-width at halfmaximum, FWHM, $0.6 \mathrm{meV}$ ) than the main line (FWHM $<0.25 \mathrm{meV}$ ), implying a singlet decay time of $1.1 \mathrm{ps}$ and that the singlet decays faster than the triplet. The different decay rates are a consequence of the spin: the triplet state has to flip a spin to relax, the singlet state does not. For the singlet, phonon scattering, which preserves electron spin, is the likely mechanism for the relaxation. Relaxation by phonon scattering is therefore fast on the timescale of recombination, a conclusion consistent with other experiments ${ }^{19,20,21}$. The argument holds also for $\mathrm{X}^{3-}$, which also shows a broad satellite PL (FWHM $1.2 \mathrm{meV}$ ).

An obvious feature in Figs 2 and 3 is the marked broadening and increase in intensity in the emission at $V_{g} \approx 0.1 \mathrm{~V}$. This looks similar to the broad emission of highly excited dots ${ }^{22}$. We suggest that the $P L$ is broad at $0.1 \mathrm{~V}$ because relaxation of the final state always proceeds quickly, regardless of spin. A new relaxation channel must open at $0.1 \mathrm{~V}$. We know from the capacitance that at $0.1 \mathrm{~V}$ the wetting layer begins to fill, and the new channel is therefore an Auger process: electrons in the quantum ring lose energy by promoting an electron in the wetting layer to an unoccupied state at higher energy ${ }^{23}$. Auger processes can swap the spins of the two participating electrons. In fact, spin-swapping processes are most likely ${ }^{24}$. This interpretation is supported by the nature of the PL at higher positive voltages, where the PL to some extent narrows again. The ring electrons can only interact with electrons in the continuum a few meV away from the Fermi energy, so that once the Fermi energy is very high, the Auger rate will decrease. The broadening at $0.1 \mathrm{~V}$ is accompanied by a rapid increase in overall intensity. This is partly caused by an increase in capture efficiency, but other factors may be involved.

\section{References}

1. Tarucha, S. , Austing, D. G. , Honda, T. , van der Hage, R. J. \& Kouwenhoven, L. P. Shell filling and spin effects in a few electron quantum dot. Phys. Rev. Lett. 77, 3613-3616 (1996).

2. Miller, B. T. et al. Few-electron ground states of charge-tunable self-assembled quantum dots. Phys. Rev. B 56, 6764- 6769 (1997).

3. Banin, U. , Cao, Y., Katz, D. \& Millo, O. Identification of atomic-like electronic states in indium arsenide nanocrystal quantum dots. Nature 400, 542-544 (1999).

4. Bimberg, D. , Grundmann, M. \& Ledentsov, N. N. Quantum Dot Heterostructures (Wiley, Chichester, 1998).

5. Yusa, G. \& Sakaki, H. Trapping of photogenerated carriers by InAs quantum dots and persistent photoconductivity in novel GaAs/n-AIGaAs field-effect transistor structures. Appl. Phys. Lett. 70, 345-347 (1997).

6. Finley, J. J. et al. Electrical detection of optically induced charge storage in self-assembled InAs quantum dots. Appl. Phys. Lett. 73, 2618-2620 (1998).

7. Lundström, T. , Schoenfeld, W. , Lee, H. \& Petroff, P. M. Exciton storage in semiconductor self-assembled quantum dots. Science 286, 2312-2314 (1999).

8. Bruchez, M. Jr, Moronne, M. , Gin, P. , Weiss, S. \& Alivisatos, A. P. Semiconductor nanocrystals as fluorescent biological labels. Science 281, 2013- 2016 (1998).

9. Garcia, J. M. et al. Intermixing and shape changes during the formation of InAs self-assembled quantum dots. Appl. Phys. Lett. 71, 2014-2016 (1997). 
10. Lorke, A. et al. Spectroscopy of nanoscopic semiconductor quantum rings. Phys. Rev. Lett. 84, 2223-2226 (2000).

11. Drexler, H. , Leonard, D., Hansen, W. , Kotthaus, J. P. \& Petroff, P. M. Spectroscopy of quantum levels in charge-tunable InGaAs quantum dots. Phys. Rev. Lett. 73, 2252-2255 (1994).

12. Ashoori, R. C. et al. N-Electron ground state energies of a quantum dot in magnetic field. Phys. Rev. Lett. 71, $613-616$ (1993).

13. Medeiros-Ribeiro, G. , Pikus, F. G. , Petroff, P. M. \& Efros, A. L. Single-electron charging and Coulomb interaction in InAs self-assembled quantum dot arrays. Phys. Rev. B 55, 1568-1573 (1997).

14. Wojs, A. \& Hawrylak, P. Theory of photoluminescence from modulation-doped self-assembled quantum dots in a magnetic field. Phys. Rev. B 55, 13066-13071 (1997).

15. Blackwood, E. , Snelling, M. J., Harley, R. T. , Andrews, S. R. \& Foxon, C. T. B. Exchange interaction of excitons in GaAs heterostructures. Phys. Rev. B 50, 14246-14254 (1994).

16. Bayer, $M$. et al. Electron and hole $g$ factors and exchange interaction from studies of the exciton fine structure in $\ln _{0.60} \mathrm{Ga}_{0.40}$ As quantum dots. Phys. Rev. Lett. 82, 1748- 1751 (1999).

17. Miller, B. T. et al. Fine structure in the spectrum of the few-electron ground states of self-assembled quantum dots. Physica B 249-251 257-261 (1998).

18. Empedocles, S. A. \& Bawendi, M. G. Quantum-confined Stark effect in single CdSe nanocrystallite quantum dots. Science 278, 2114-2117 (1997).

19. Gammon, D. , Snow, E. S. , Shanabrook, B. V., Katzer, D. S. \& Park, D. Homogeneous linewidths in the optical spectrum of a single gallium arsenide quantum dot. Science 273, 87-90 (1996).

20. Sosnowski, T. S. et al. Rapid carrier relaxation in $\ln _{0.4} \mathrm{Ga}_{0.6} \mathrm{As} / \mathrm{GaAs}$ quantum dots characterized by differential transmission spectroscopy. Phys. Rev. B 57, R9423-R9426 (1998).

21. Toda, Y., Moriwaki, O. , Nishioka, M. \& Arakawa, Y. Efficient carrier relaxation mechanism in InGaAs/GaAs self-assembled quantum dots based on the existence of continuum states. Phys. Rev. Lett. 82, 4114-4117 ( 1999).

22. Deckel, E. et al. Multiexciton spectroscopy of a single self-assembled quantum dot. Phys. Rev. Lett. 80, 4991- 4994 (1998).

23. Bockelmann, U. et al. Time resolved spectroscopy of single quantum dots: Fermi gas of excitons? Phys. Rev. Lett. 76, 3622- 3625 (1996).

24. Ridley, B. K. Quantum Processes in Semiconductors (Clarendon, Oxford, 1982).

\section{Acknowledgements}

We thank J. P. Kotthaus and N. D. Drew for discussions. This work was supported by the Deutsche Forschungsgemeinschaft and by QUEST, an NSF Science and Technology Center. 